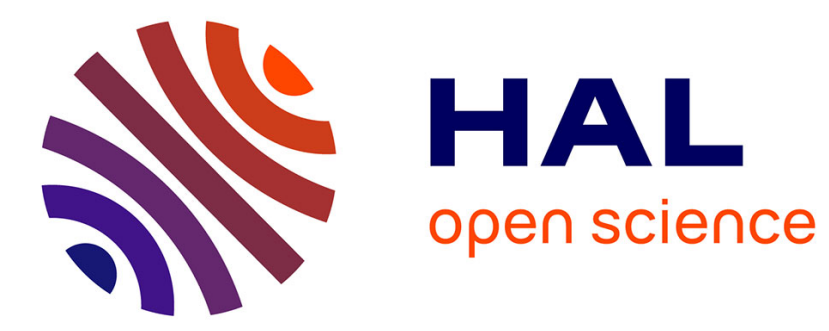

\title{
Anisotropic relativistic cross sections for inelastic electron scattering, and the magic angle
}

Peter Schattschneider, Cécile Hébert, Henri Franco, Bernard Jouffrey

\section{To cite this version:}

Peter Schattschneider, Cécile Hébert, Henri Franco, Bernard Jouffrey. Anisotropic relativistic cross sections for inelastic electron scattering, and the magic angle. Physical Review B: Condensed Matter and Materials Physics (1998-2015), 2005, 72, pp.045142 (1-8). 10.1103/PhysRevB.72.045142 . hal00014441

\section{HAL Id: hal-00014441 \\ https://hal.science/hal-00014441}

Submitted on 9 Dec 2005

HAL is a multi-disciplinary open access archive for the deposit and dissemination of scientific research documents, whether they are published or not. The documents may come from teaching and research institutions in France or abroad, or from public or private research centers.
L'archive ouverte pluridisciplinaire HAL, est destinée au dépôt et à la diffusion de documents scientifiques de niveau recherche, publiés ou non, émanant des établissements d'enseignement et de recherche français ou étrangers, des laboratoires publics ou privés. 


\title{
Anisotropic relativistic cross sections for inelastic electron scattering and the magic angle
}

\author{
P. Schattschneider, C. Hébert, H. Franco \\ Inst. f. Festkörperphysik, TU Wien, Wiedner Hauptstraße 8-10, A-1040 WIEN, Austria \\ B. Jouffrey \\ Laboratoire MSS-Mat, École Centrale Paris, Grande Voie des Vignes, 92295 Châtenay Malabry Cedex
}

(Dated: November 25, 2005)

\begin{abstract}
The knowledge of the inelastic double differential scattering cross section (DDSCS) is of utmost importance for microanalysis in Electron Energy Loss Spectrometry (EELS) in the electron microscope and for the investigation of electron energy loss near edge structure (ELNES). Calculations are usually based on a non-relativistic first order Born approximation. Relativistic corrections were thought to be important only for primary beam energies much higher than $300 \mathrm{keV}$. A semirelativistic correction taking into account the relativistic mass change of the probe electron was considered sufficient ${ }^{1}$. We review the relativistic expression of the inelastic double differential scattering cross section (DDSCS) originally given by Møller ${ }^{2}$. We show that the virtual photon exchange ${ }^{3}$ need not be considered separately. The interference terms between longitudinal and transverse exitations cannot be neglected in anisotropic systems. This explains the surprisingly small magic angle.
\end{abstract}

\section{INTRODUCTION}

The recent progress in instrumentation has made Electron Energy Loss Spectrometry (EELS) in the Transmission Electron Microscope (TEM)an important method of solid state spectrometry. Spectrometers or energy filters of the last generation in combination with monochromators and field emission sources allow the study of the electronic structure of materials with unprecedented accuracy. Of prime interest in this context are low energy losses and the energy loss near edge structure of ionization edges (ELNES). The fine structure can now be measured with an energy resolution of $\sim 0.1 \mathrm{eV}$, comparable to what synchrotrons provide, but on a length scale of nanometers.

Although the anisotropic electronic structure was investigated very early with EELS ${ }^{4}$ the method was only used extensively when parallel recording spectrometers bacame available, and when the ELNES could be calculated with sufficient accuracy ${ }^{5-8}$.

The local environment of an ionized atom defines its electronic structure and thus its ELNES, allowing identification of phases by fingerprinting ( $i$. e. comparison with ELNES spectra of standard specimens). A typical example is the carbon $\mathrm{K}$-edge ELNES in various carbon containing compounds. The fundamental question is then if there exist experimental conditions where fingerprinting would not be superimposed or made impossible by anisotropy effects. The same problem occurs in the determination of the $\mathrm{sp} 2 / \mathrm{sp} 3$ ratio in various modifications of carbon, in particular in diamondlike amorphous carbon $^{9,10}$

Therefore much work has been devoted to the determination of experimental conditions where the effect of anistropy is cancelled $d^{5,11-16}$. A simple geometric reasoning shows that these conditions are realized when a particular collection angle for the inelastically scat- tered electrons is chosen The angle at which the effects of anisotropy are cancelled was called magic angle as a referring to X Ray absorption spectroscopy.

The theoretical values found for the magic angle in these works varies from $1.36 \vartheta_{e}$ (Paxton et al.) to $4 \vartheta_{e}$ (Souche et al. and Menon et al.) Daniels et al. found a theoretical value of $2 \vartheta_{e}$ but their derivation was shown to contain an error - the correct value is also $4 \vartheta_{e}^{14}$. Paxton et al. ${ }^{12}$ claimed to find $1.36 \vartheta_{e}$ but their calculation must contain a trivial mistake since the theoretical derivation is correct and gives also $4 \vartheta_{e}$.

The only detailed experimental work was published by Daniels et al. ${ }^{13}$. Their finding (with a magic angle close to $2 \vartheta_{e}$ ) was verified independently ${ }^{14}$.

These recent experiments suggested that theory must have missed an essential point. Various possibilities were proposed such as neglect of non-dipole transitions, intensities coming from other Bragg spots or channeling effects ${ }^{14}$

Recently the weak point in theory was discovered ${ }^{14}$. It could be shown that the usual relativistic correction based on the papers by Møller, Bethe, Fano, repeated in textbooks and used in numerous studies of the DDSCS applies only to isotropcic systems. In anisotropic systems, the corrections for the contributions parallel and perpendicular to the incident electrons' trajectory must be treated separately.

The very reason for this unexpected behaviour is the relativistic change of the electic field of the fast probe electron. The electric field of a moving charge is no longer spherically symmetric but "'compressed"' in the direction of motion ${ }^{17}$. As a consequence, the Coulomb coupling $\propto q^{-4}$ occurring in the double differential inelastic scattering cross section (DDSCS) becomes anisotropic. The coupling to transitions with momentum transfer parallel to the electrons' trajectory becomes fainter, and the coupling to transitions perpendicular to the trajectory becomes stronger. Since these changes act in opposite 
ways, their effect on the magic angle is dramatic.

These findings were published in a seminal short paper $^{15}$. The derivation given there could have been considered incomplete for two reasons: first, reference was made to an article by Bethe written in German ${ }^{18}$, without giving any details; second, only the Lorentz transform of the momentum transfer $\hbar \mathbf{q}$ from the probe electron's frame to the rest frame of the scatterer was considered. The virtual photon exchange at relativistic speeds described by Fano $^{3}$ was not taken into account explicitely. $\mathrm{In}^{15}$ it was speculated that the remaining discrepancies between theory and experiment could be explained by this transverse virtual photon exchange. But there was also indications that the inclusion of this effect yields the same result ${ }^{19}$.

In the present paper we put the previous findings onto firm ground by following the original approach of $\mathrm{M} \varnothing \mathrm{ller}^{2}$ in a form adapted to a beam of unpolarized probe electrons. We can then discard the electron spin and need not use Dirac spinors. This simplification makes the derivations much shorter and facilitates the understanding of the underlying physics. We shall see that the expression for the scattering amplitude given by Fano - including transverse photon exchange - is identical to our previous one, and no further correction need be done.

On the other hand, Fano's statement that the longitudinal and transverse contributions to the DDSCS can be added incoherently - which can be traced back to the work of Bethe ${ }^{20}$ and has been repeated several times ${ }^{21,22}$ - turns out to be wrong for anisotropic systems. This surprising discovery bears important consequences on the investigation of relativistic DDSCSs of anisotropic systems, including reliable values for the calculation of ELNES. In the end, Fano's statement seems to have been at the very heart of the magic angle problem.

\section{FULLY RELATIVISTIC BETHE THORY}

In this section, we derive a relativistic expression for the DDSCS based on Bethe's theory ${ }^{18}$ for inelastic electron scattering. We express the perturbing potential in the transition matrix element by the electromagnetic scalar and vector potentials of the fast probe electron. The advantage of the present approach is that contrary to the original work of Møller ${ }^{2}$ we do not need Dirac matrices or spinors, thus clarifying the underlying physical principles. Still the result is exact in first order for non magnetic materials.

In first order plane wave Born approximation the double differential scattering cross section (DDSCS) for inelastic electron scattering on matter reads ${ }^{23}$

$\frac{\partial^{2} \sigma}{\partial E \partial \Omega}=\left(\frac{2 \pi}{\hbar}\right)^{4} \gamma^{2} m^{2} \sum_{i, f} \frac{k_{f}}{k_{i}}\left|\left\langle f\left|\left\langle k_{f}|V| k_{i}\right\rangle\right| i\right\rangle\right|^{2} \delta\left(E_{a}-E_{b}\right)$

where $m$ is the electron mass, $\left|k_{i, f}\right\rangle$ are plane waves describing the incident and outgoing probe electron, and $|i\rangle$, $|f\rangle$ are initial and final states of the scatterer connected by the transition induced by the perturbation. $E_{a, b}$ are the energies of the closed system before and after scattering, and $V$ is the perturbation in the Hamiltonian of the system. In the following, we restrict ourselves to a oneelectron model, that is we assume that only one electron of the scatterer is involved in a transition at a time. This is a good approximation whenever correlation is a minor effect or can be treated approximately, such as in DFT theory for inner shell ionization, or in the low energy loss region when the joint density of states is an appropriate model for intra- and interband transitions.

The unperturbed Hamiltonian of the scatterer with eigenfunctions $|i\rangle,|f\rangle$ is

$$
H_{0}=\frac{\hat{\mathbf{p}}^{2}}{2 m}+e V_{0}
$$

where $\hat{\mathbf{p}}$ is the momentum operator and $V_{0}$ is the self consistent potential of the scatterer. We shall use the "hat" symbol for operators only in cases where confusion with 3 - or 4-vectors could render the reading more difficult.

The probe electron moving at speed $\mathbf{v}_{0}$ adds scalar and vector potentials $\Phi, \mathbf{A}$ to the system. The perturbed Hamiltonian is

$$
H=\frac{1}{2 m}\left(\hat{\mathbf{p}}-\frac{e}{c} \mathbf{A}\right)^{2}+e\left(V_{0}+\Phi\right) .
$$

Comparison of Eqs.2 and 3 shows that the perturbation is $^{29}$

$$
V=e \Phi-\frac{e}{m c} \hat{\mathbf{p}} \mathbf{A}+\frac{e^{2}}{2 m c^{2}} \mathbf{A}^{2}
$$

Although the electric and magnetic fields responsible for the perturbation are gauge invariant it is convenient to use the Lorentz gauge since the differential equations for the potentials are then Lorentz invariant ${ }^{24}$. In this case the vector potential relates to the scalar one by

$$
\mathbf{A}=\frac{\mathbf{v}_{0}}{c} \Phi
$$

Inserting Eq.5 into Eq.4, and retaining only the leading terms up to order $c^{-2}$ gives

$$
V=e \Phi\left(1-\frac{\hat{\mathbf{p}} \mathbf{v}_{0}}{m c^{2}}\right)
$$

for the perturbation in first order Born approximation. Note that $V$ is a function of the distance between probe electron at position $\mathbf{r}$ and target electron at $\mathbf{R}$.

The matrix element $\left\langle k_{i}|V| k_{f}\right\rangle$ can now be calculated, using the shift theorem for Fourier transforms, as

$\left\langle k_{i}|V| k_{f}\right\rangle=\frac{1}{(2 \pi)^{3}} \int d^{3} r e^{i \mathbf{q r}} V(\mathbf{r}-\mathbf{R})=\frac{1}{(2 \pi)^{3}} V(\mathbf{q}) e^{i \mathbf{q} \mathbf{R}}$.

where $V(\mathbf{q})$ is the Fourier component of the perturbation for wave vector $\mathbf{q}=\mathbf{k}_{i}-\mathbf{k}_{f}$. In the Lorentz gauge, the 
scalar potential $\Phi$ of the probe electron obeys the field equation

$$
\Delta \Phi-\frac{1}{c^{2}} \frac{\partial^{2} \Phi}{\partial t^{2}}=-4 \pi \rho .
$$

In the rest frame of the scatterer, $\rho=e \delta^{3}\left(\mathbf{r}-\mathbf{v}_{0} t\right)$ represents the moving electron.

In order to solve Eq.8 we switch to the $(\mathbf{q}, \omega)$ representation: Here, the field equation becomes a simple algebraic equation

$$
\left(q^{2}-\frac{\omega^{2}}{c^{2}}\right) \Phi=-4 \pi e \delta\left(\omega-\mathbf{q v}_{0}\right)
$$

with solution

$$
\Phi=\frac{-4 \pi e \delta\left(\omega-\mathbf{q} \mathbf{v}_{0}\right)}{q^{2}-\frac{\omega^{2}}{c^{2}}}
$$

The term $\omega^{2} / c^{2}$ in the denominator is a direct consequence of retardation in the relativistic description of the Coulomb interaction. This term is responsible for the increased Coulomb coupling. The delta function relates a particular energy loss $E=\hbar \omega$ of the incident electron to a momentum transfer

$$
\hbar q_{z}=E / v_{0}
$$

that is, it selects a characteristic (and minimum) momentum transfer in the transition. For convenience we have defined a coordinate system with the z-axis parallel to the incident particle's trajectory $\mathbf{v}_{0}$. Inserting Eqs.10, 7 and 6 into Eq.1 we obtain for the DDSCS

$$
\frac{\partial^{2} \sigma}{\partial E \partial \Omega}=\left[\left(\frac{2 \gamma m e^{2}}{\hbar^{2}}\right)\right]^{2} \frac{1}{\left(q^{2}-\omega^{2} / c^{2}\right)^{2}} \frac{k_{f}}{k_{i}} \sum_{i, f} \mid\langle f| e^{i \mathbf{q} \hat{\mathbf{R}}}\left(1-\frac{\hat{\mathbf{p}} \mathbf{v}_{0}}{m c^{2}}\right)
$$

The delta function still describes energy conservation of the closed system. Its argument has been rewritten such that the energy loss $E$ of the probe electron occurs explicitely. It must equal the energy gain $E_{f}-E_{i}$ of the scatterer. The relationship between $\mathbf{q}$ and the scattering angle is given by the scattering geometry

$$
q^{2}=k_{i}^{2}+k_{f}^{2}-2 k_{i} k_{f} \cos \vartheta
$$

For small $\vartheta$, the cosine can be expanded to quadratic order, and with $k_{i} \approx k_{f}$ we obtain

$$
q^{2} \doteq k_{i}^{2} \vartheta^{2}+q_{z}^{2}
$$

For medium acceleration voltages $<400 \mathrm{keV}$ the observable momentum transfers in inelastic electron scattering are $<\sim 10^{9} / \mathrm{m}$. In this momentum range $q a_{0} \ll 1$, so the dipole approximation is valid. This simplifies expression 12 further. Expanding the operator the leading terms (linear in $\mathrm{R}$ ) are

$$
e^{i \mathbf{q} \hat{\mathbf{R}}}\left(1-\frac{\hat{\mathbf{p}} \mathbf{v}_{0}}{m c^{2}}\right) \doteq 1+i \mathbf{q} \hat{\mathbf{R}}-\frac{\hat{\mathbf{p}} \mathbf{v}_{0}}{m c^{2}}-i \mathbf{q} \hat{\mathbf{R}} \frac{\hat{\mathbf{p}} \mathbf{v}_{0}}{m c^{2}}
$$

The constant term does not contribute to the matrix element according to orthogonality of the initial and final states. Observing

$$
\frac{1}{m}\langle i|\hat{\mathbf{p}}| f\rangle=i \omega_{i f}\langle i|\hat{\mathbf{R}}| f\rangle
$$

the fourth term is of order $R^{2}$ and will be neglected. In other words, the dipole approximation corresponds to taking only the second and third term in Eq.13 when calculating the matrix element of Eq.12. With the boundary condition Eq.11 this is

$$
\left\langle i\left|i \mathbf{q} \hat{\mathbf{R}}-i \omega_{i f} \frac{\hat{\mathbf{R}} \mathbf{v}_{0}}{c^{2}}\right| f\right\rangle=i\left(\mathbf{q}-q_{z} \mathbf{e}_{z} \frac{v_{0}^{2}}{c^{2}}\right)\langle i|\hat{\mathbf{R}}| f\rangle
$$

We can bring eq.12 in a final form. Inserting the matrix element eq.15, and the boundary condition eq.11 we obtain

$$
\frac{\partial^{2} \sigma}{\partial E \partial \Omega}=\frac{4 \gamma^{2}}{a_{0}^{2} Q^{2}} \frac{k_{f}}{k_{i}} S\left(\mathbf{q}^{\prime}, E\right)
$$

with $^{30}$

$$
\mathbf{q}=\left(\begin{array}{l}
q_{x} \\
q_{y} \\
q_{z}
\end{array}\right)
$$

$$
\mathbf{q}^{\prime}=\mathbf{q}-\left(\begin{array}{c}
0 \\
0 \\
q_{z} \beta^{2}
\end{array}\right)
$$

and the abbreviation

$$
Q=q^{2}-q_{z}^{2} \beta^{2}
$$

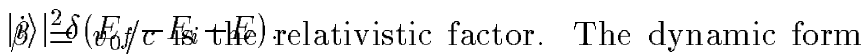
factor (DFF) in dipole approximation is

$$
S_{d i p}\left(\mathbf{q}^{\prime}, E\right)=\sum_{i, f}\left|\left\langle f\left|\mathbf{q}^{\prime} \hat{\mathbf{R}}\right| i\right\rangle\right|^{2} \delta\left(E_{f}-E_{i}-E\right) .
$$

This is exactly the equation given by Møller ${ }^{2}$, but for non-magnetic materials.

Some comments are in place on Eq. 16. The prefactor has decreased, that is the DDSCS has increased. This was due to the use of retarded potentials. One also remarks that the denominator is a relativistic 4-scalar and as such Lorentz invariant ${ }^{25}$. The argument of the DFF has also decreased but only for transitions with q vectors in z-direction. The combined effect tends to increase the DDSCS for scattering angles close to

$$
\vartheta_{e}=\frac{q_{z}}{k_{i}}=\frac{E}{2 \gamma T} .
$$

where $T$ is the classical kinetic energy of the probe electron. For isotropic systems, $S_{d i p} \propto{q^{\prime}}^{2}$ we obtain

$$
\frac{\partial^{2} \sigma}{\partial E \partial \Omega} \propto \frac{q_{x}^{2}+q_{z}^{2}\left(1-\beta^{2}\right)^{2}}{\left(q_{x}^{2}+q_{z}^{2}\left(1-\beta^{2}\right)\right)^{2}} \propto \frac{\vartheta^{2}+\vartheta_{e}^{2}\left(1-\beta^{2}\right)^{2}}{\left(\vartheta^{2}+\vartheta_{e}^{2}\left(1-\beta^{2}\right)\right)^{2}}
$$


This function is shown in fig. 1 for $100,200,400$, and $1000 \mathrm{keV}$, scaled to $\vartheta_{e}$. At high energy the DDSCS has a dip in forward direction. Such behaviour was predicted on the basis of dielectric theor $\mathrm{y}^{26}$ and experimentally confirmed $^{27}$.

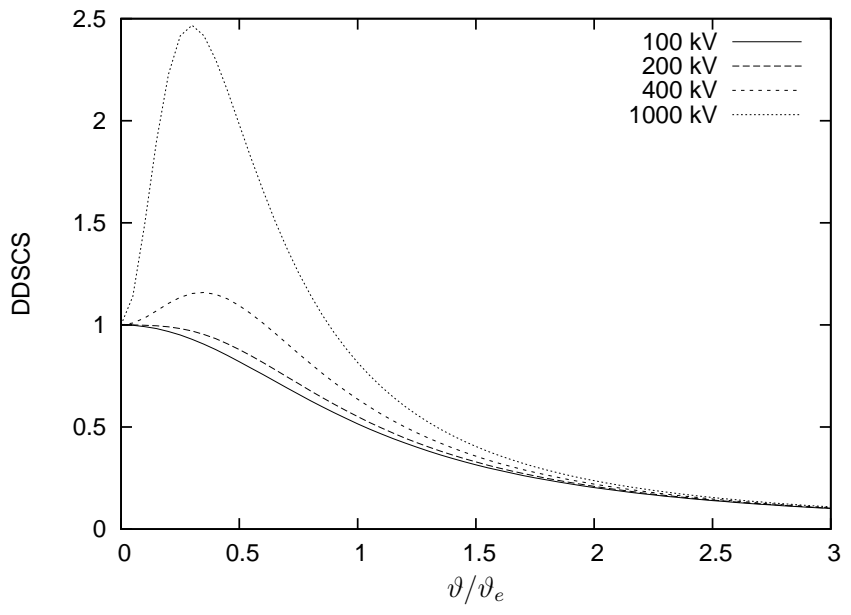

FIG. 1: DDSCS as a function of incident energy (from bottom to top $100,200,400,1000 \mathrm{keV})$. Abscissa in units $\vartheta_{e}$.

\section{THE VIRTUAL PHOTON EXCHANGE}

We can rewrite Eq.16 as

$$
\frac{\partial \sigma}{\partial E \partial \Omega}=\sum_{i, f}\left(\frac{2 \gamma}{a_{0}}\right)^{2}\left|\left(\frac{\mathbf{q}-\beta \frac{\omega}{c} \mathbf{e}_{\mathbf{z}}}{q^{2}-\left(\frac{\omega}{c}\right)^{2}}\right)\langle i|\mathbf{R}| f\rangle\right|^{2}
$$

which is exactly the form given by Møller.

We separate the term containing $\mathbf{e}_{z}$ into longitudinal and transverse components where the "transverse" unit polarization vector $\mathbf{t}$ of the virtual photon is perpendicular to the "longitudinal" momentum transfer, $\mathbf{t} \perp \mathbf{q}$.

$$
\mathbf{e}_{z}=\frac{\mathbf{e}_{z} \mathbf{q}}{q^{2}} \mathbf{q}+\mathbf{e}_{z} \mathbf{t}
$$

After minor algebra eq.21 reads

$$
\frac{\partial \sigma}{\partial E \partial \Omega}=\sum_{i, f}\left(\frac{2 \gamma}{a_{0}}\right)^{2}\left|\left(\frac{\mathbf{q}}{q^{2}}-\frac{\beta \frac{\omega}{c} \mathbf{e}_{\mathbf{z}} \cdot \mathbf{t}}{q^{2}-\left(\frac{\omega}{c}\right)^{2}} \mathbf{t}\right)\langle i|\mathbf{R}| f\rangle\right|^{2}
$$

When we replace the prefactor by

$$
\left(\frac{2 \gamma}{a_{0}}\right)^{2}=\left(\frac{e^{2}}{\hbar c}\right)^{2} \frac{4 k^{2}}{\beta^{2}}
$$

we obtain the expression given by $\mathrm{Fano}^{3}$

The first term is a pure Coulomb interaction term, i.e. longitudinal and not retarded. The relativistic effect is visible as an additional term that can be interpreted as the exchange of a virtual photon with polarization perpendicular to q. In fact, this form is obtained directly when instead of the Lorentz gauge the Coulomb gauge is used for the potentials. The equivalence that we proved above is a consequence of gauge invariance. The expression given in $^{15}$ is correct. We have thus excluded that virtual photon exchange can explain the remaining discrepancies between theory and experiment.

\section{THE MAGIC ANGLE}

We generalize here the derivation given in ${ }^{15}$ for uniaxial anisotropy. With the transition matrix

$$
\begin{gathered}
\hat{\mathbf{R}}=\left\{R_{j l}\right\} \\
R_{j l}:=\sum_{i, f}\left\langle i\left|R_{j}\right| f\right\rangle\left\langle f\left|R_{l}\right| i\right\rangle \delta\left(E-E_{i}+E_{f}\right)
\end{gathered}
$$

the DFF Eq.19 is

$$
S_{d i p}\left(\mathbf{q}^{\prime}, E\right)=\mathbf{q}^{\prime} \hat{\mathbf{R}} \mathbf{q}^{\prime}
$$

It can be shown that under very general conditions ${ }^{31}$ the transition matrix is diagonal in its natural coordinate system (i. e. where the scatterer has the highest symmetry)

$$
\hat{\mathbf{R}}=\boldsymbol{\Lambda}\left(R_{j j}\right)
$$

We note in passing that $S_{d i p}$ is a quadratic form.

check the consistent use of $q^{\prime}$ (the relativ. corrected q) and $x y z$ instead of $x 1,2,3$

By definition, the magic angle is that collection angle $\Theta$ at which the partial cross section

$$
\Delta \sigma(\Gamma, \Theta, E)=\frac{4 \gamma^{2}}{a_{0}^{2}} \frac{k_{f}}{k_{i}} \int_{0}^{2 \pi} d \varphi \int_{0}^{\Theta} \frac{S\left(\mathbf{q}^{\prime}, E\right)}{Q^{2}} \vartheta d \vartheta
$$

is independent of the specimen's orientation. Let $\Gamma$ be the tilt of the axis $R_{3}$ with respect to the incident electron's trajectory $z$ over axis $x$, and let the angle between the axes $R_{1}$ and $x$ be $\Psi$. The rotation relating the two coordinate systems is then described by the unitary matrix

$$
\hat{\mathbf{U}}=\left(\begin{array}{ccc}
\cos \Psi & -\cos \Gamma \sin \Psi & \sin \Gamma \sin \Psi \\
\sin \Psi & \cos \Gamma \cos \Psi & -\cos \Psi \sin \Gamma \\
0 & \sin \Gamma & \cos \Gamma
\end{array}\right)
$$

In the laboratory system $\{x, y, z\}$ the transition matrix reads

$$
\hat{\mathbf{R}}=\hat{\mathbf{U}}(\Gamma)^{-1} \boldsymbol{\Lambda}\left(R_{i i}\right) \hat{\mathbf{U}}(\Gamma) .
$$

Preparing for the angular integrations over azimuth $\phi$ and scattering angle $\vartheta$, we use the momentum transfer $\mathbf{q}$ from Eq.18 in the small angle approximation

$$
\mathbf{q}^{\prime}=\left(\begin{array}{c}
k_{i} \vartheta \sin \varphi \\
k_{i} \vartheta \cos \varphi \\
k_{i} \vartheta_{e} / \gamma^{2}
\end{array}\right)
$$


With Eqs. 27 and 28 the DFF, Eq.24 can be expressed in terms of angles $\varphi, \vartheta, \Gamma$. With these prerequisites, the $\varphi$ integral in the partial cross section Eq.26 can be performed first. The terms containing $\sin \varphi, \cos \varphi$ or $\sin \varphi \cos \varphi$ vanish according to orthogonality. After some algebra we obtain

$$
\begin{aligned}
\Delta \sigma(\Gamma, \Theta, E)= & \frac{4 \gamma^{2} \pi}{a_{0}^{2} k_{i}^{2}} \frac{k_{f}}{k_{i}} \\
& \left\{R_{11}\left[a\left(\cos ^{2} \Gamma \sin ^{2} \Psi+\cos ^{2} \Psi\right)+c \sin ^{2} \Gamma \sin ^{2} \Psi\right]_{5}\right. \\
+ & R_{22}\left[a\left(\cos ^{2} \Gamma \cos ^{2} \Psi+\sin ^{2} \Psi\right)+c \sin ^{2} \Gamma \cos ^{2} \Psi\right] 0 \\
+ & \left.R_{33}\left[c \cos ^{2} \Gamma+a \sin ^{2} \Gamma\right]\right\}
\end{aligned}
$$$$
\left\{R_{11}\left[a\left(\cos ^{2} \Gamma \sin ^{2} \Psi+\cos ^{2} \Psi\right)+c \sin ^{2} \Gamma \sin ^{2} \Psi\right]\right]_{5}
$$

with

$$
\begin{aligned}
& a(\Theta):=\int_{0}^{\Theta} \frac{\vartheta^{3}}{\left(\vartheta^{2}+\vartheta_{e}^{2} / \gamma^{2}\right)^{2}} d \vartheta \\
& c(\Theta):=2 \int_{0}^{\Theta} \frac{\vartheta_{e}^{2} \vartheta}{\left(\vartheta^{2}+\vartheta_{e}^{2} / \gamma^{2}\right)^{2} \gamma^{4}} d \vartheta
\end{aligned}
$$

The result has a remarkable structure. It is a linear combination of the three transition matrix elements $R_{i i}$ with $\Gamma$ - and $\Psi$-dependent coefficients. The factors $a$ and $c$ can be interpreted as the coupling constants of the probe electron to transitions with momentum transfers perpendicular and parallel to the trajectory. For $\Gamma=0$ these coincide with transitions involving matrix elements $R_{11}$ (or $R_{22}$ ) and $R_{33}$.

In the non-relativistic limit, $Q \rightarrow q^{2}, \gamma \rightarrow 1$. It is evident from Eq. 30 that the relativistic correction increases the coupling by the common denominator $\left(\vartheta^{2}+\vartheta_{e}^{2} / \gamma^{2}\right)^{2}$, but the coupling to transitions $R_{33}$ is diminished by the relativistic factor $\gamma^{-4}$ which counteracts the first effect.

Expression 29 is independent of the tilt angles $\Psi, \Gamma$ if $R_{11}=R_{22}=R_{33}$ or if

$$
a=c \text {. }
$$

The first condition means isotropy and need not be discussed further. The second condition defines the magic angle. Fig. 2 shows the functions $c$ (thick line) and $a$ (thin line) as a function of $\Theta$, for $200 \mathrm{keV}$ incident energy. The crossing of the curves defines the magic angle. The solution of Eq. 31 turns out to be a function of $\vartheta / \vartheta_{e}$, so the abscissa is given in units of $\vartheta_{e}$ here and in the following.

In the non-relativistic limit, $Q \rightarrow q^{2}, \gamma \rightarrow 1$ we obtain the dashed lines. Now the reason for the dramatic effect of the relativistic correction is visible: it has diminished the coupling constant $\mathrm{c}$ and increased the constant $\mathrm{a}$, thus changing the crossing of the curves significantly.

Fig. 3 shows the magic angle as a function of incident energy. At $200 \mathrm{keV}$ it is found numerically to be $1.46 \vartheta_{e}$. The non-relativistic value is $3.97 \vartheta_{e}$

The table gives values obtained by solving Eq.31 with the Newton algorithm.

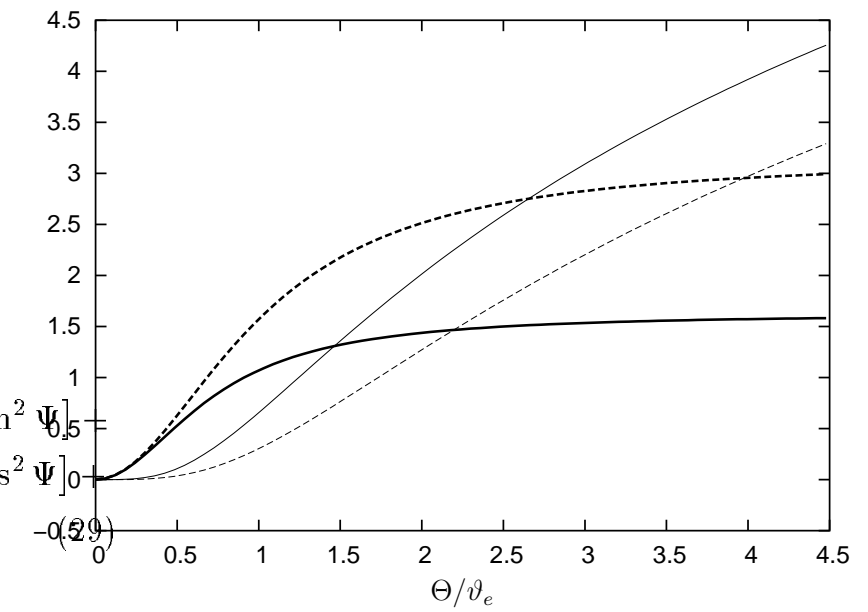

FIG. 2: Prefactors a and c of the functions $R_{11}$ and $R_{33}$, Eq. 30. thick lines: c, thin lines a. The crossing defines the magic angle. Dashed: non-relativistic factors. Abscissa in units $\vartheta_{e}$.

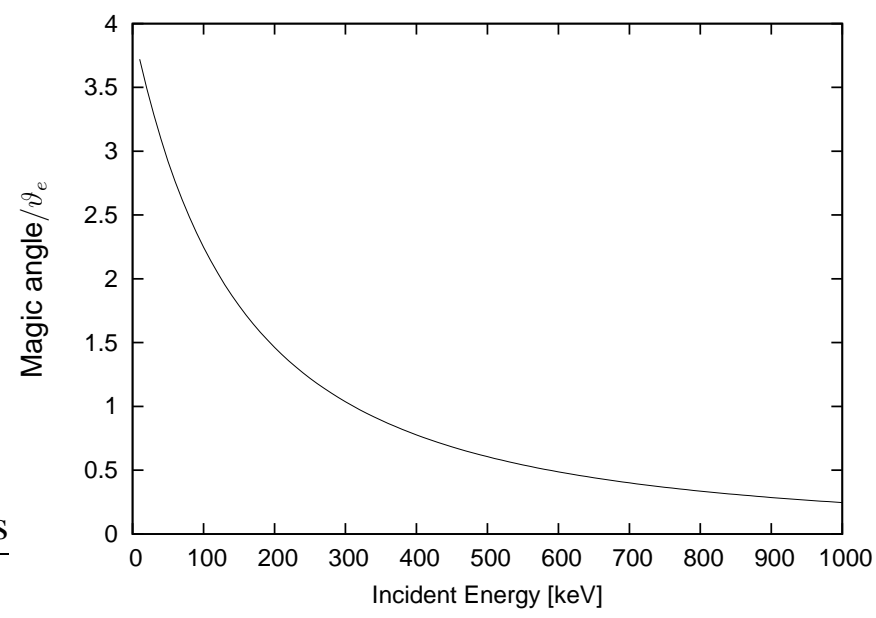

FIG. 3: Magic angle (in units $\vartheta_{e}$ ) as a function of incident energy. Abscissa in keV.

\section{INCOHERENT SUMMATION}

Fano $^{3}$ argues that in the Coulomb gauge the longitudinal and transverse terms can be added incoherently since they belong to different selection rules. In fact, if a longitudinal perturbation in direction $\mathrm{z}$, say, induces an $\mathrm{s} \rightarrow \mathrm{p}_{\mathrm{z}}$ transition, then the transverse component is ineffective for this transition (it rather induces $s \rightarrow p_{x}$ ).

A closer inspection shows however that this is only true for isotropic systems. Indeed, in the Coulomb gauge the matrix element of Eq.22 can be rewritten

$$
\left(\frac{\mathbf{q}}{q^{2}}-\frac{\beta \frac{\omega}{c} \mathbf{e}_{\mathbf{z}} \cdot \mathbf{t}}{q^{2}-\left(\frac{\omega}{c}\right)^{2}} \mathbf{t}\right)\langle i|\mathbf{R}| f\rangle=\frac{1}{Q}(\mathbf{q}-C \mathbf{t})\langle i|\mathbf{R}| f\rangle .
$$




\begin{tabular}{c|cc}
$E_{0}[\mathrm{keV}]$ & $\gamma$ & magic angle $\left[\vartheta_{e}\right]$ \\
\hline 0 & 1. & 3.98 \\
50 & 1.09785 & 2.92 \\
100 & 1.19569 & 2.25 \\
150 & 1.29354 & 1.790 \\
200 & 1.39139 & 1.46 \\
250 & 1.48924 & 1.22 \\
300 & 1.58708 & 1.04 \\
350 & 1.68493 & 0.89 \\
400 & 1.78278 & 0.78 \\
500 & 1.97847 & 0.61 \\
1000 & 2.95695 & 0.25
\end{tabular}

The DFF, Eq.24 is then

$S_{d i p}\left(\mathbf{q}^{\prime}, E\right)=(\mathbf{q}-C \mathbf{t}) \hat{\mathbf{R}}(\mathbf{q}-C \mathbf{t})=\mathbf{q} \hat{\mathbf{R}} \mathbf{q}+C^{2} \mathbf{t} \hat{\mathbf{R}} \mathbf{t}-2 C \Re[\mathbf{q} \hat{\mathbf{R}} \mathbf{t}]$

The first term is the non-relativistic DFF in dipole approximation, the second one is the relativistic correction from the exchange of the virtual photon, and the last term describes interference between the two terms. It is, in general, not zero since the vector $\hat{\mathbf{R}} \mathbf{t}$ is not perpendicular to q. Only when the matrix elements $R_{i i}$ are all equal this term vanishes according to the orthogonality of $\mathbf{q}$ and $\mathbf{t}$.

The statement of Fano corresponds to the DFF

$$
S_{\text {Fano }}\left(\mathbf{q}^{\prime}, E\right)=\mathbf{q} \hat{\mathbf{R}} \mathbf{q}+\mathbf{t} \hat{\mathbf{R}} \mathbf{t}
$$

that is, neglecting the interference term. This expression is correct for isotropic systems. $\mathbf{t} \hat{\mathbf{R}} \mathbf{t}$ is the only correction term.

When we calculate the prefactors a and c with Eq.34 we obtain the results shown in fig. 4 . The magic angle is now $4.27 \vartheta_{e}$.

(The results shown in figs. 4 to 7 relate to an incident energy of $200 \mathrm{keV}$ ).

Obviously this approach to the calculation of the magic angle is wrong. Referring to Eq.19 the DDSCS can be separated into terms stemming from transitions involving the operators $\hat{x}, \hat{y}$ and $\hat{z}$. When the specimen is oriented with its axes $R_{1}, R_{2}, R_{3}$ parallel to the laboratory system $x, y, z$ the cross terms between $x y, x z, y z$ vanish. The factors $\mathrm{a}$ and $\mathrm{c}$ can then be interpreted as the angular integrals over the DDSCSs for transitions involving operators $\hat{x}$ and $\hat{y}$ perpendicular to the incident beam direction, and $\hat{z}$ parallel to the incident beam. The ELNES of the graphite $\mathrm{K}$ edge is a good example (assuming that at given energy loss only $\sigma^{*}$ or $\pi^{*}$ final states are available). For uniaxial anisotropic systems, $|\langle\hat{x}\rangle|^{2}=|\langle\hat{y}\rangle|^{2} \neq|\langle\hat{z}\rangle|^{2}$ where $\vec{x}$ is some general position vector in the $\mathrm{x}-\mathrm{y}$ plane, we can define two DDDSCSs

$$
\begin{gathered}
\sigma_{\perp}=\frac{4 \gamma^{2}}{a_{0}^{2} Q^{2}} \frac{k_{f}}{k_{i}}\left(q_{x}^{2}+q_{y}^{2}\right)|\langle\hat{x}\rangle|^{2} \\
\sigma_{\|}=\frac{4 \gamma^{2}}{a_{0}^{2} Q^{2}} \frac{k_{f}}{k_{i}}{q_{z}^{\prime 2}}_{z}^{2}|\langle\hat{z}\rangle|^{2}
\end{gathered}
$$

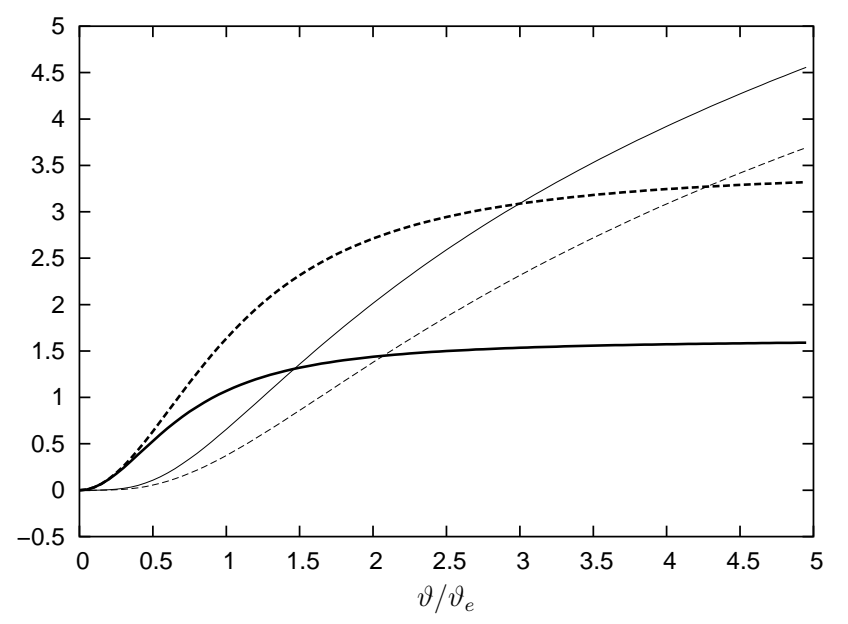

FIG. 4: Full lines: prefactors a and c, Eq. 30 compared with calculations neglecting interference, based on Eq.34 (dashed), for $200 \mathrm{keV}$ incident energy. thick lines: c, thin lines: a. The crossing defines the magic angle which is $4.27 \vartheta_{e}$ in the Fano approach. Abscissa in units $\vartheta_{e}$.

They are shown in fig.5, normalized to 1 . They can be visualized as angular scattering distributions when the scatterer allows only transitions $s \rightarrow p_{x}$, or $s \rightarrow p_{z}$ with p-orbitals oriented parallel to the respective axes of the laboratory frame, i, e. $p_{z}$ has its axis parallel to the incident electron trajectory. $\sigma_{\perp}$ is dashed, $\sigma_{\|}$is dotted. The thin lines are non-relativistic values, i. .e. the first term in Eq.34, the thick lines include the second term. It seems that the corrections are indeed small. The full lines show the sum of $\sigma_{\perp}$ and $\sigma_{\|}$. This sum correctly describes the relativistic correction for isotropic systems, as already shown in fig.1.

The interference term $-2 C \mathbf{q} \hat{\mathbf{R}} \mathbf{t}$ in Eq.33 is shown in fig. 6, again separated into perpendicular and zcomponents. The terms are of order $20 \%$, with a maximum attained below the magic angle. The terms have equal value, but opposite sign.

It is the very interference that explains the small magic angle. Fig.7 shows $\sigma_{\perp}$ and $\sigma_{\|}$with and without interference term.

Without interference terms, all corrections are positive, and the magic angle changes only slightly. The 


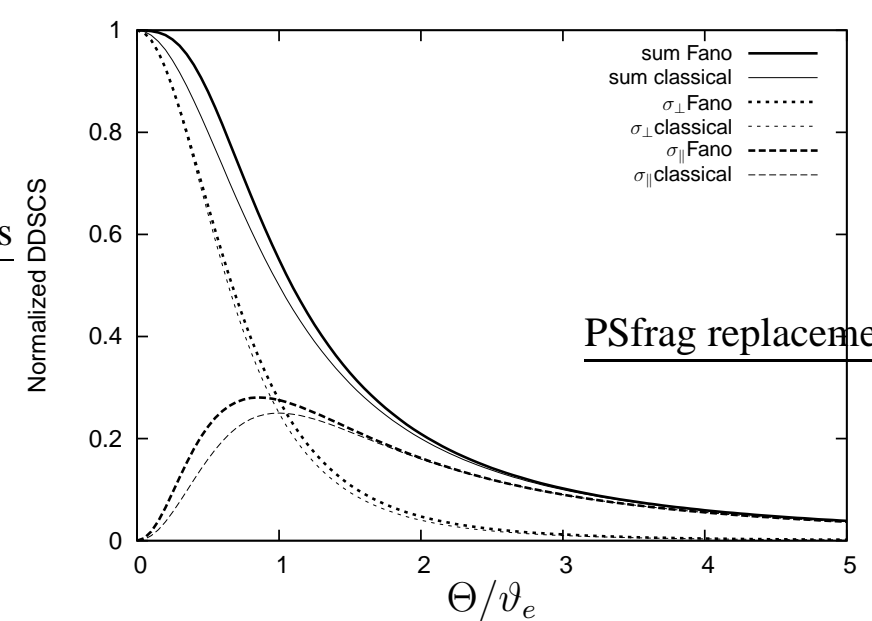

FIG. 5: Normalized DDSCS, Eqs.35, $36 \sigma_{\perp}$ (dotted) and $\sigma_{\|}$ (dashed), and their sum (full lines). Thin lines are nonrelativistic, thick lines in the Fano approximation, Eq.34. Abscissa is in units of $\vartheta_{e}$.

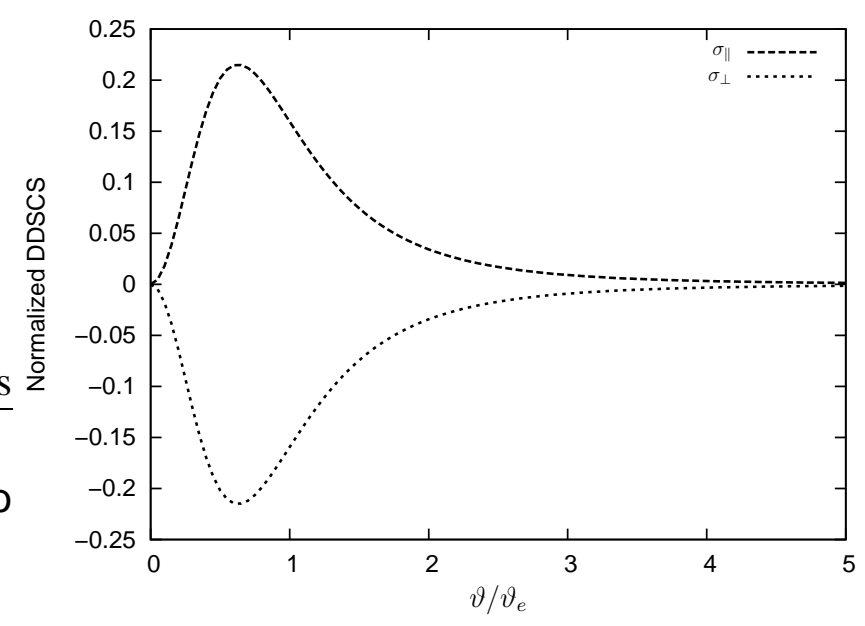

FIG. 6: Interference term for $\sigma_{\|}$(dashed) and $\sigma_{\perp}$ (dotted). Abscissa in units $\vartheta_{e}$

correct treatment including interference causes a strong negative correction for $\sigma_{\perp}$ and an even stronger positive correction for $\sigma_{\|}$. The angular integrals become then equal at much smaller $\Theta$. Interference explains also why in isotropic systems the correction to the DDSCS is small: In the sum over $\sigma_{\perp}$ and $\sigma_{\|}$the corrections with different sign cancel completely.

The relativistic correction in the Coulomb gauge allows another interesting insight: it acts as an additive term in the amplitude, of order $\sim \beta E / \hbar c \sim q_{z} \beta^{2}$ (see the boundary condition for $q_{z}$, Eq. 11). Therefore the interference term is of order $\sim \beta^{2}$ whereas the direct correction term is of order $\sim \beta^{4}$. It is only due to the fact that the interference term vanishes for isotropic systems that the relativistic correction is only of order $\sim \beta^{4}$. But

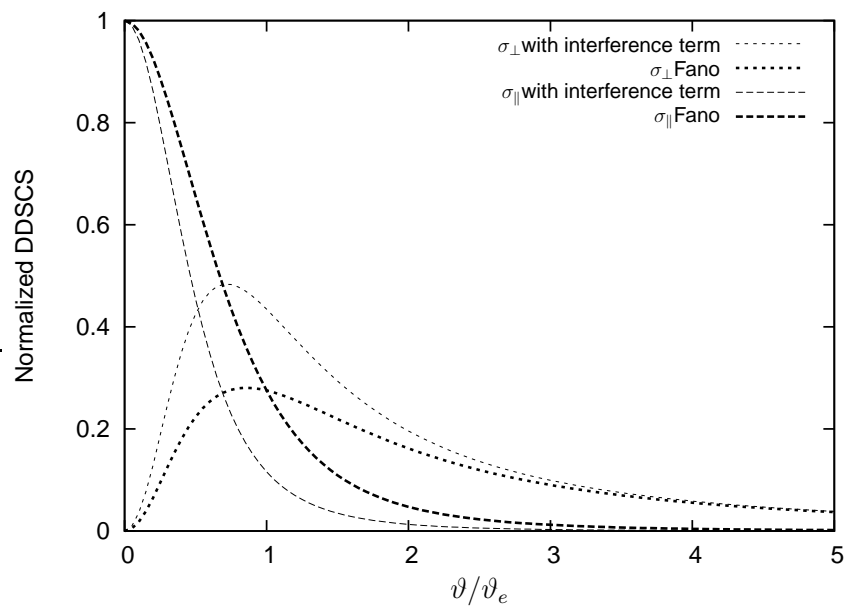

FIG. 7: Comparison of normalized DDSCSs for $\sigma_{\perp}$ (dotted) and $\sigma_{\|}$(dashed). Thick lines are Fano-corrected (Eq.34), thin lines exact expression including interference terms. The perpendicular component is increased, the parallel component is decreased.

for anisotropic ones - and this is most surprising - it is of order $\sim \beta^{2}$.

It can be cautiously presumed that some relativistic corrections published in earlier work are not reliable.

For instance, the meticulous experimental work of Daniels et. al. ${ }^{13}$ suggested nearly the same magic angle for 100 and for $200 \mathrm{keV}$ but this may have been biased by their erroneous theoretical premise and the considerable experimental noise. To finally clarify the situation it is therefore mandatory to perform experiments at various incident energies under the same geometry and with significantly reduced noise. Work is in progress on this subject.

It also means that the semi-relativistic or classical approximations used for the calculation of ELNES (which were thought to be sufficient according to the negligible relativistic effect in the medium voltage range) do not hold for anisotropic systems. A thorough recalculation will probably show some unexpected result.

\section{SEMI-RELATIVISTIC CORRECTION OF $\vartheta_{e}$}

In the limit $c \rightarrow \infty$ Eq.12reduces immediately to the non-relativistic expression for the scattering cross section $^{18,23}$. A well known form is

$$
\frac{\partial^{2} \sigma}{\partial E \partial \Omega}=\left[\frac{2 m e^{2}}{(\hbar q)^{2}}\right]^{2} \frac{k_{f}}{k_{i}} S(\mathbf{q}, E)
$$

The often applied semi relativistic correction accounts only for the effect of the relativistic increase of mass on the characteristic scattering angle. This angle $\vartheta_{e}$ 
defines the FWHM of the angular scattering distribution for given energy loss. In the dipole approximation, $S(\mathbf{q}, E) \propto q^{2}$, so the scattering distribution is a Lorentzian in $q_{x}$,

$$
\frac{\partial^{2} \sigma}{\partial E \partial \Omega} \propto \frac{1}{q_{z}^{2}+q_{x}^{2}}
$$

and the FWHM is defined by

$$
\begin{gathered}
q_{x}=q_{z} \\
\vartheta_{e}=q_{x} / k_{i}=q_{z} / k_{i}=\Delta p / p_{i}
\end{gathered}
$$

where $\Delta p$ is the momentum transferred in an exact forward scattering event. For small energy losses, $E \ll E_{0}$

$$
\left.\Delta p \doteq \frac{d p}{d E}\right|_{E_{0}} E .
$$

The kinetic energy for fast particles $E_{0}=p_{0}^{2} /(2 \gamma m)$ deviates from the classical quadratic $p^{2}$ behaviour $^{3}$, and

$$
\frac{d E}{d p}=\frac{p}{\gamma m_{0}}=v .
$$

Therefore

$$
\vartheta_{e}=\frac{\Delta p}{p_{0}}=\frac{E}{p_{0} v_{0}}=\frac{E}{\gamma m v_{0}^{2}} .
$$

or $^{1} \vartheta_{e}=E /(2 \gamma T)$ with the classical kinetic energy $T=m v_{0}^{2} / 2$ of the incident electron. This is a good approximation for incident energies up to $300 \mathrm{keV}$.

It should be noted that the relativistically corrected characteristic angle stems from the scattering kinematics which are different for fast particles according to the relativistic mass increase. They even apply to the non-relativistic cross section, Eq.37. According to the Lorentzian character of the angular distribution, $\vartheta_{e}$ serves as a scaling unit. It is often convenient to use dimensionless scattering angles $\vartheta / \vartheta_{e}$. In principle, both the classical and the relativistic scaling angles can be used. It is however more reasonable to use the latter one since it defines the experimental FWHM. We have used this unit in all calculations.

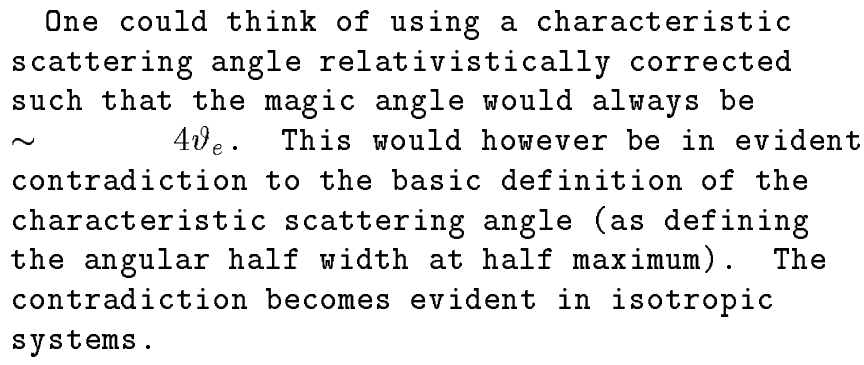

\section{CONCLUSION}

A thorough derivation of relativistic corrections to the DDSCS in first order plane wave Born approximation, based on the relativistically correct scalar and vector potential of the fast probe electron occurring in the interaction Hamiltonian shows that the virtual photon exchange $^{3}$ need not be considered separately. It only occurs in the Coulomb gauge. In the Lorentz gauge used in our previous theory ${ }^{15}$ it is implicitely included.

Interference terms play an important role and turn out to be of order $\beta^{2}$ for anisotropic systems, acting oppositely for transitions involving momentum transfers parallel and perpendicular to the probe electron's trajectory. This explains the small magic angle found experimentally. We showed that the standard corrections of order $\beta^{4}$ routinely applied in EELS are only valid for isotropic systems whereas in anisotropic ones the correction is of order $\beta^{2}$. This finding bears important consequences for the correct interpretation of ELNES in anisotropic systems and may explain previous discrepancies between theory and experiment.

Acknowledgements: Part of this work was sponsored by the European Union, under FP6-2003-NEST-A, contract nr. 508971 (CHIRALTEM)
1 R. F. Egerton, Electron Energy Loss Spectroscopy in the electron microscope (Plenum Press, xx, 1986).

2 C. Møller, Annalen der Physik 14, 531 (1932).

3 U. Fano, Physical Review 102, 385 (1956).

${ }^{4}$ K. Zeppenfeld, Zeitschrift für Physik 243, 229 (1971).

${ }^{5}$ C. Souche, B. Jouffrey, G. Hug, and M. Nelhiebel, Micron 29, 419 (1998).

${ }^{6}$ C. Hébert-Souche et al., Ultramicroscopy 83, 9 (2000).

7 K. Lie, R. Høier, and R. Brydson, Physical Review B 61, 1786 (2000).

8 D. Su, C. Hébert, M. Willinger, and R. Schlögl, Micron 34, 227 (2003).

9 S. D. Berger, D. R. McKenzie, and P. J. Martin, Philo- sophical Magazine Letters 57, 285 (1988).

10 Y. Wang, Ultramicroscopy 33, 151 (1990).

11 N. K. Menon and J. Yuan, Ultramicroscopy 74, 83 (1998).

12 A. T. Paxton, M. Vanschilfgaarde, M. Mackenzie, and A. J. Craven, Journal of Physics: Condensed Matter 12, 729 (2000).

13 H. Daniels et al., Ultramicroscopy 96, 523 (2003).

14 C. Hébert, P. Schattschneider, and B. Jouffrey, Ultramicroscopy 101, 271 (2004).

15 B. Jouffrey, P. Schattschneider, and C. Hébert, Ultramicroscopy 102, 61 (2004).

16 Y. K. Sun and J. Yuan, Condensed Matter, abstract xx, http://arxiv.org/abs/cond (2004). 
17 J. Jackson, Classical electrodynamics (xx, yy, 1962).

18 H. A. Bethe, Annalen der Physik 5, 325 (1930).

19 Y. K. Sun and J. Yuan, Condensed Matter, abstract http://arxiv.org/abs/cond (2004).

${ }^{20} \mathrm{H}$. Bethe, in Quantentheorie, edited by A. Smekal (xx, yy, 1933), pp. 495-506.

21 R. Anholt, Physical review A 19, 1004 (nd).

22 R. Knippelmeyer and H. Kohl, Journal of Microscopy 194, 30 (1999).

23 P. Schattschneider, Fundamentals of inelastic electron scattering (Springer, New York, 1986).

${ }^{24}$ L. D. Landau and E. M. Lifshitz, Quantum mechanics,
Non-relativistic Theory (xx, yy, 1977).

25 R. J. Gould, Physica 62, 555 (1972).

${ }^{26}$ E. Kröger, Zeitschrift für Physik 216, 115 (1968).

27 H. Kurata, P. Wahlbring, S. Isoda, and T. Kobayashi, Micron 28, 381 (1997).

28 M. Nelhiebel et al., Physical Review B 59, 12807 (1999).

29 Since $\mathbf{A}$ and $\mathbf{p}$ commute.

${ }^{30}$ In ref. ${ }^{15}$, there is a mistake in Q: instead of q', there we must have $\mathrm{q}$.

31 When the scatterer has at least two perpendicular mirror planes and is non-magnetic ${ }^{28}$. 